\title{
REMOÇÃO DE COR EM SOLUÇÕES DE CORANTES REATIVOS POR OXIDAÇÃO COM $\mathrm{H}_{2} \mathrm{O}_{2} / \mathrm{UV}$
}

Fabiana Valéria da Fonseca Araujo* e Lídia Yokoyama

Departamento de Processos Inorgânicos, Escola de Química, Universidade Federal do Rio de Janeiro, Bloco E, Cid. Univ. Ilha do Fundão, 21949-900 Rio de Janeiro - RJ

Luiz Alberto César Teixeira

Departamento de Ciências dos Materiais e Metalurgia, Pontifícia Universidade Católica, 22453-900 Rio de Janeiro - RJ

Recebido em 6/7/04; aceito em 16/6/05; publicado na web em 01/12/05

COLOR REMOVAL IN REACTIVE DYE SOLUTIONS BY UV/H $\mathrm{O}_{2}$ OXIDATION. This paper summarizes the result of a degradation test of two azo-reactive dyes (Reactive Blue 214, Reactive Red 243) under UV irradiation in the presence of $\mathrm{H}_{2} \mathrm{O}_{2}$. Five different doses of hydrogen peroxide $(0 \mathrm{mM}, 5 \mathrm{mM}, 10 \mathrm{mM}, 20 \mathrm{mM}$ and $30 \mathrm{mM})$ at constant initial concentration of the substrate $(100 \mathrm{mg} / \mathrm{L})$ were used. The radiation source were three $15 \mathrm{~W}$-lamps. Complete destruction of the color of the solutions was attained in 40-50 min of irradiation. $\mathrm{UV} / \mathrm{H}_{2} \mathrm{O}_{2}$ proved capable of complete discoloration and degradation of the above azo reactive dyes.

Keywords: reactive dyes; hydrogen peroxide; UV radiation.

\section{INTRODUÇÃO}

A indústria têxtil gera efluentes com composição extremamente heterogênea e uma grande quantidade de material tóxico e recalcitrante, o que torna seu tratamento mais difícil. Esses efluentes apresentam uma forte coloração, uma grande quantidade de sólidos suspensos, $\mathrm{pH}$ altamente flutuante, temperatura elevada, grandes concentrações de DQO, considerável quantidade de metais pesados (ex. $\mathrm{Cr}$, Ni ou $\mathrm{Cu}$ ), compostos orgânicos clorados e surfactantes ${ }^{1}$.

Os corantes reativos são amplamente utilizados na indústria têxtil devido a sua boa estabilidade durante a lavagem e por apresentarem procedimentos simples de tingimento, sendo a principal classe de corantes utilizada para tingir celulose e algodão ${ }^{2}$. Entretanto, esses corantes são altamente solúveis em água e ainda apresentam baixos níveis de fixação nas fibras, sendo em boa parte perdidos no efluente.

A estrutura dos corantes reativos apresenta pelo menos um grupamento cromóforo constituído pela ligação azo $(\mathrm{N}=\mathrm{N})$, principal responsável pela cor do tingimento ${ }^{3}$. A coloração presente no efluente descartado pelas indústrias têxteis é reduzida com tratamentos que levam à clivagem das ligações azo.

Os processos mais comuns empregados para tratamento de efluentes têxteis são os biológicos e físico-químicos. No entanto, estudos realizados têm mostrado que os corantes reativos, devido a sua complexa estrutura química, são resistentes à degradação biológica, dificultando a remoção de cor dos efluentes ${ }^{4-6}$. Os processos físico-químicos, como coagulação/floculação, adsorção com carvão ativado e membranas têm sido desenvolvidos para remover a cor em efluentes têxteis, entretanto, essas tecnologias somente transferem o corante de fase, não resolvendo essencialmente o problema ${ }^{7,8}$.

Alguns autores têm estudado a utilização de um tratamento fotoeletroquímico para remoção de cor em efluentes têxteis com corantes reativos. Esse processo poderia ser utilizado em uma etapa posterior ao tratamento biológico, apenas para remoção da cor? .

Devido às limitações das tecnologias convencionais para desco-

*e-mail: fabianavf@ig.com.br loração de efluentes têxteis com corantes reativos, tem se buscado desenvolvimento de tecnologias efetivas, técnica e economicamente, para resolução do impasse ambiental. Deste modo, a utilização de Processos Oxidativos Avançados (POAs) tem se tornado uma alternativa potencial para reduzir a cor desses efluentes, que geralmente está relacionada à presença de substâncias recalcitrantes.

Os POAs são tecnologias que geralmente utilizam um forte agente oxidante $\left(\mathrm{O}_{3}, \mathrm{H}_{2} \mathrm{O}_{2}\right)$ e/ou catalisadores $\left(\mathrm{Fe}, \mathrm{Mn}, \mathrm{TiO}_{2}\right)$ na presença ou não de fonte de irradiação, para gerar radicais livres $\mathrm{OH} \bullet\left(\mathrm{E}^{0}=2,80 \mathrm{~V}\right)$, altamente reativos, capazes de mineralizar substâncias orgânicas refratárias, presentes nos efluentes industriais ${ }^{10}$.

Um método direto para geração de $\mathrm{OH} \bullet$ é a fotoclivagem do peróxido de hidrogênio por meio de irradiação UV. O processo $\mathrm{H}_{2} \mathrm{O}_{2} / \mathrm{UV}$ pode levar à degradação completa e à conversão $\mathrm{CO}_{2} \mathrm{e}$ $\mathrm{H}_{2} \mathrm{O}$ e sais inorgânicos da maioria dos contaminantes orgânicos.

A fotólise do $\mathrm{H}_{2} \mathrm{O}_{2}$ pela radiação UV gera dois radicais hidroxila (reação 1), que agem degradando a matéria orgânica e formando compostos mais simples (reação 2). Quando o $\mathrm{H}_{2} \mathrm{O}_{2}$ está em excesso pode ocorrer uma reação paralela, que diminui a razão de degradação da matéria orgânica (reação 3). Isso ocorre porque $\mathrm{o}_{2} \mathrm{H}_{2} \mathrm{O}_{2}$ em excesso age capturando radicais hidroxilas.

$\mathrm{H}_{2} \mathrm{O}_{2}+\mathrm{hv} \rightarrow 2 \mathrm{OH} \bullet$

$\mathrm{R}-\mathrm{H}+\mathrm{OH} \bullet \rightarrow$ Produtos finais

(reação 1)

$\left(\mathrm{CO}_{2}, \mathrm{H}_{2} \mathrm{O}, \mathrm{NO}_{3}^{-}, \mathrm{Cl}^{-}\right)$ (reação 2)

$\mathrm{OH} \bullet+\mathrm{H}_{2} \mathrm{O}_{2} \rightarrow \mathrm{HO}_{2} \bullet+\mathrm{H}_{2} \mathrm{O}$ (reação 3)

O objetivo deste estudo foi avaliar a degradação dos corantes reativos Azul Marinho Drimarene X-GN 150 (C. I Reactive Blue 214) e Vermelho Drimarene X-6BN 150 (C.I. Reactive Red 243) através da utilização do sistema $\mathrm{H}_{2} \mathrm{O}_{2} / \mathrm{UV}$.

\section{PARTE EXPERIMENTAL}

\section{Materiais}

Os corantes Azul Marinho Drimarene X-GN 150 e Vermelho Drimarene X-6BN-150 (Figuras 1 e 2), obtidos sob forma de pó, 
são produzidos pela Clariant. Soluções aquosas contendo $100 \mathrm{mg} / \mathrm{L}$ dos corantes foram preparadas com água destilada.

O peróxido de hidrogênio $(30 \% \mathrm{p} / \mathrm{p})$, gentilmente fornecido pela Peróxidos do Brasil Ltda, foi adicionado em quantidades préestabelecidas de forma a se obter concentrações em solução de 5, 10,20 e $30 \mathrm{mM}$

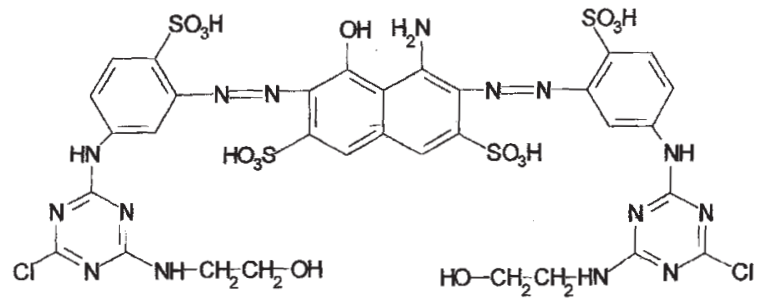

Figura 1. Estrutura do corante Azul Marinho Drimarene X-GN 150 (C.I Reactive Blue 214)

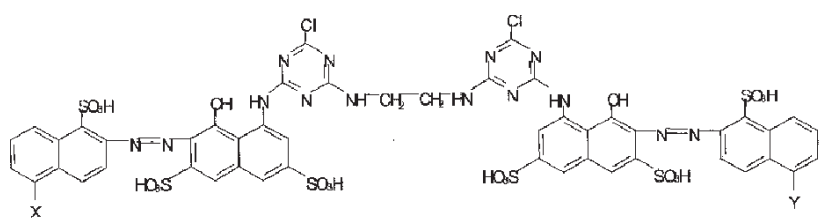

Mistura de 3 moléculas : tipo 1: $X=Y=\mathrm{SO}_{3} \mathrm{H}$; tipo 2: $X=\mathrm{SO}_{3} \mathrm{H}, \mathrm{Y}=\mathrm{H}$; tipo $3: \mathrm{X}=\mathrm{Y}=\mathrm{H}$

Figura 2. Estrutura do corante Vermelho Drimarene X-6BN 150 (C.I Reactive Red 243)

\section{Aparato experimental}

$\mathrm{O}$ equipamento utilizado para tratamento fotoquímico (Figura 3) foi constituído para processar $300 \mathrm{~mL}$ de solução, em sistema de batelada.

Durante os experimentos, a solução a ser tratada foi mantida em um recipiente de vidro, sob agitação e na ausência de luz externa.

A incidência da radiação UV foi feita na superfície da solução, a partir de três lâmpadas de vapor de mercúrio (Germetec) de $30 \mathrm{~cm}$, cada uma com potência nominal de $15 \mathrm{~W}$, a uma distância de $15 \mathrm{~cm}$ da solução.

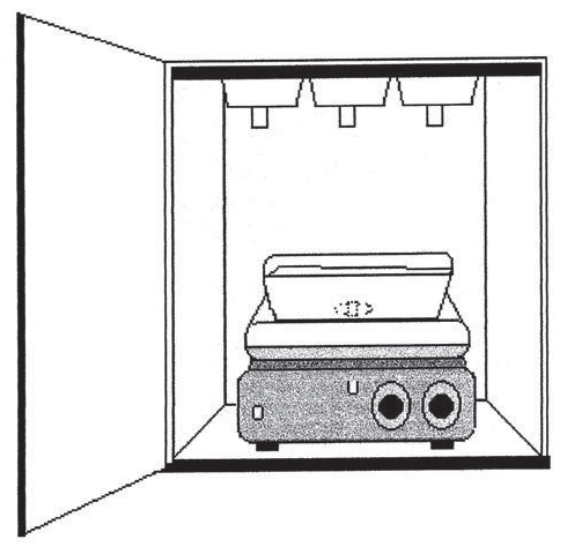

Figura 3. Aparato experimental usado nos experimentos

\section{Metodologia}

Os experimentos de fotodegradação foram realizados à temperatura de $298 \mathrm{~K}$ e o tempo final de reação foi de $60 \mathrm{~min}$.

A reação iniciava-se pela adição do $\mathrm{H}_{2} \mathrm{O}_{2}(0,5,10,20$ e $30 \mathrm{mM})$ à solução de $100 \mathrm{mg} / \mathrm{L}$ do corante reativo, e posterior ligação das lâmpadas UV.

A descoloração das soluções dos corantes reativos foi acompanhada através das medições de absorbância em espectrofotômetro Shimadzu modelo UV-Mini - 1240, usando comprimento de onda de $613 \mathrm{~nm}$ para corante Azul Drimarene X-GN e $516 \mathrm{~nm}$ para corante Vermelho Drimarene X-6BN. Esses valores correspondem àqueles comprimentos de onda para os quais se observou maior absorbância.

A demanda química de oxigênio foi medida através do método espectrofotométrico com prévia digestão, conforme descrito no Standard Methods - seção 5220 D ${ }^{11}$.

\section{RESULTADOS E DISCUSSÃO}

As Figuras 4 e 5 apresentam a evolução dos valores da absorbância das soluções dos corantes reativos em diferentes concentrações de $\mathrm{H}_{2} \mathrm{O}_{2}$ durante o tratamento fotoquímico. É interessante observar que na ausência de $\mathrm{H}_{2} \mathrm{O}_{2}(0 \mathrm{mM})$ não ocorre mudança na absorbância das soluções em 60 min de tratamento.

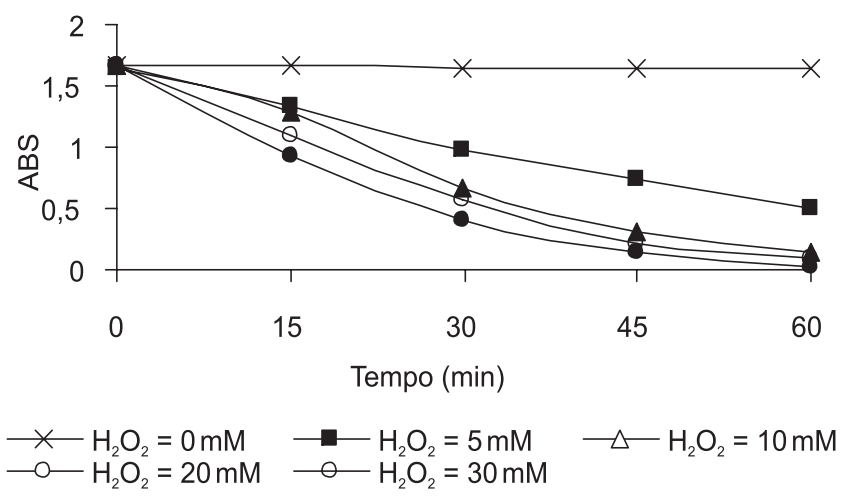

Figura 4. Testes de irradiação UV do corante Azul Marinho X-GN $150 \mathrm{com}$ várias concentrações de $\mathrm{H}_{2} \mathrm{O}_{2}$

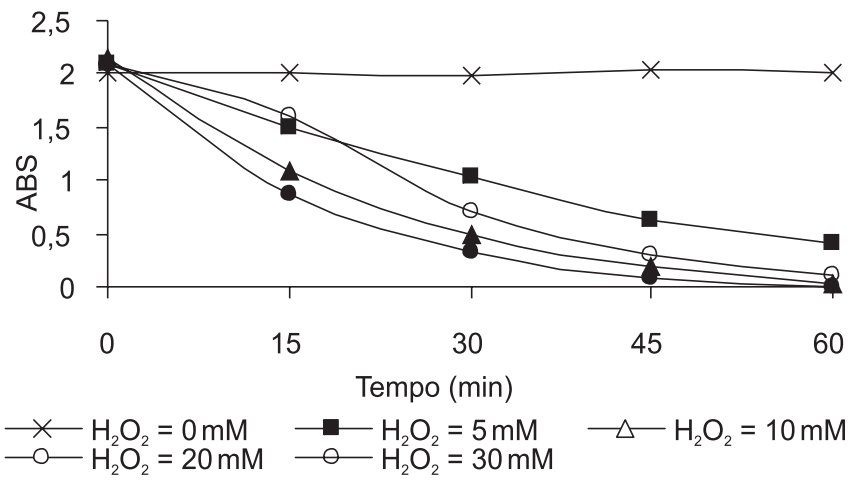

Figura 5. Testes de irradiação UV do corante Vermelho Drimarene X-6BN 150 com várias concentrações de $\mathrm{H}_{2} \mathrm{O}_{2}$

A combinação de peróxido de hidrogênio com a radiação UV é necessária para produzir radicais $\mathrm{OH} \bullet$ e iniciar a reação de oxidação da ligação azo reduzindo, assim, a presença de cor nas soluções. O poder oxidante do peróxido de hidrogênio não foi suficien- 
te para promover a degradação dos corantes reativos, necessitando da presença de irradiação UV para que ocorra a degradação dos corantes. A razão de descoloração é favorecida pela presença de $\mathrm{H}_{2} \mathrm{O}_{2}$, devido aos radicais hidroxila gerados durante as reações fotoquímicas ${ }^{12}$. Os resultados também mostraram a dependência da razão de descoloração dos corantes Vermelho Drimarene X-6BN 150 e Azul Marinho Drimarene X-GN 150 com a concentração inicial de peróxido de hidrogênio.

A degradação do corante, e conseqüente remoção de cor na solução, é aumentada consideravelmente com o aumento da concentração inicial de $\mathrm{H}_{2} \mathrm{O}_{2}$ de 0 para $20 \mathrm{mM}$. Entretanto, elevandose a concentração inicial de peróxido de hidrogênio de 20 para $30 \mathrm{mM}$ pôde-se observar uma significante diminuição no desempenho do processo $\mathrm{H}_{2} \mathrm{O}_{2} / \mathrm{UV}$, provavelmente, devido à competição do $\mathrm{H}_{2} \mathrm{O}_{2}$ que age "capturando" radicais reativos hidroxila $\mathrm{HO} \bullet$ para formar um radical menos reativo $\mathrm{HO}_{2} \bullet$. Isso significa que acima de $20 \mathrm{mM}$ de $\mathrm{H}_{2} \mathrm{O}_{2}$ há consumo do peróxido de hidrogênio, para formação de $\mathrm{HO}_{2} \bullet$ (reação 3).

A concentração de corante na solução foi reduzida em até $98 \%$ para o corante Azul e 99,8\% para o corante Vermelho utilizando-se uma concentração de $\mathrm{H}_{2} \mathrm{O}_{2}$ de $20 \mathrm{mM}$ após 60 min de reação, como pode ser visto nas Tabelas 1 e 2. Esses resultados evidenciam uma completa degradação dos corantes reativos testados pelo sistema $\mathrm{H}_{2} \mathrm{O}_{2} / \mathrm{UV}$.

Tabela 1. Concentração final do corante reativo Azul Drimarene X-GN 150 na solução para diferentes $\mathrm{C}_{\mathrm{H}_{2} \mathrm{O}_{2}} \cdot \mathrm{C}_{\mathrm{A}}{ }^{0}=100 \mathrm{mg} / \mathrm{L}$

\begin{tabular}{lrrrrr}
\hline $\begin{array}{l}\text { Tempo } \\
\text { (min) }\end{array}$ & $\mathbf{0}$ & \multicolumn{1}{c}{$\mathrm{C}_{\mathrm{H}_{2} \mathrm{O}_{2}}(\mathrm{mM})$} & \multicolumn{1}{c}{$\mathbf{1 0}$} & \multicolumn{1}{c}{$\mathbf{2 0}$} & \multicolumn{1}{c}{$\mathbf{3 0}$} \\
\hline 0 & 100,00 & 100,00 & 100,00 & 100,00 & 100,00 \\
15 & 100,00 & 79,32 & 76,38 & 54,99 & 66,21 \\
30 & 99,82 & 58,40 & 39,54 & 23,90 & 26,68 \\
45 & 99,84 & 44,26 & 18,44 & 8,07 & 10,44 \\
60 & 99,82 & 29,32 & 8,17 & 1,73 & 3,60 \\
\hline
\end{tabular}

Tabela 2. Concentração final do corante reativo Vermelho Drimarene $\mathrm{X}-6 \mathrm{BN} 150$ na solução para diferentes $\mathrm{C}_{\mathrm{H} 2 \mathrm{O} 2} \cdot \mathrm{C}_{\mathrm{A}}{ }^{0}$ $=100 \mathrm{mg} / \mathrm{L}$

\begin{tabular}{lrrrrr}
\hline $\begin{array}{l}\text { Tempo } \\
\text { (min) }\end{array}$ & $\mathbf{0}$ & \multicolumn{1}{c}{$\mathrm{C}_{\mathrm{H}_{2} \mathrm{O}_{2}}(\mathrm{mM})$} \\
\hline 0 & 100,00 & 100,00 & \multicolumn{1}{c}{100,00} & \multicolumn{1}{c}{$\mathbf{2 0}$} & \multicolumn{1}{c}{$\mathbf{3 0}$} \\
15 & 98,46 & 65,94 & 47,76 & 38,50 & 100,00 \\
30 & 97,98 & 44,82 & 20,98 & 13,95 & 30,31 \\
45 & 97,06 & 27,05 & 8,00 & 3,20 & 12,69 \\
60 & 96,78 & 17,68 & 1,75 & 0,20 & 4,75 \\
\hline
\end{tabular}

Para uma interpretação quantitativa dos resultados foi estabelecido um estudo do modelo cinético. A cinética de degradação dos corantes reativos pode ser expressa pela Equação 1

$-\frac{d C A}{d \tau}=\mathrm{K}_{2} \mathrm{C}_{\mathrm{A}} \mathrm{C}_{\mathrm{HO}}$

onde, $\mathrm{C}_{\mathrm{A}}$ representa a concentração do corante azo e $\mathrm{C}_{\mathrm{HO}}$. a concentração do radical hidroxila. Em concordância com Neamtu et al. ${ }^{12}$, pode-se considerar que a $\mathrm{C}_{\mathrm{HO}}$. é uma constante, quando em presença de excesso de peróxido de hidrogênio. Neste caso, a Equação 1 pode ser simplificado em um modelo cinético de pseudo-primeira ordem (Equação 2) $-\frac{d C A}{d \tau}=\mathrm{K}_{1} \mathrm{C}_{\mathrm{A}}$

As constantes de pseudo-primeira ordem foram calculadas usando-se regressão linear a partir dos dados experimentais apresentados nas Tabelas 1 e 2 . Os resultados calculados são apresentados na Tabela 3.

Tabela 3. Constantes de pseudo-primeira ordem dos corantes reativos Azul Drimarene XGN-150 e Vermelho Drimarene X-6BN 150

\begin{tabular}{lcc}
\hline & \multicolumn{2}{c}{$\mathrm{K}_{1}\left(\mathrm{~min}^{-1}\right)$} \\
$\mathrm{C}_{\mathrm{H}_{2} \mathrm{O}_{2}}^{0}(\mathrm{mM})$ & $\begin{array}{c}\text { Azul Drimarene } \\
\text { XGN-150 }\end{array}$ & $\begin{array}{c}\text { Vermelho Drimarene } \\
\text { X-6BN } 150\end{array}$ \\
\hline 0,0 & 0,00003 & 0,00055 \\
5,0 & 0,02045 & 0,02888 \\
10,0 & 0,04175 & 0,06743 \\
20,0 & 0,06762 & 0,10358 \\
30,0 & 0,05540 & 0,05078 \\
\hline
\end{tabular}

A razão de degradação aumenta consideravelmente com o aumento da concentração inicial de $\mathrm{H}_{2} \mathrm{O}_{2}$ de 0 para $5 \mathrm{mM}$. Entretanto, a dosagem de oxidante foi aumentada gradualmente até um certo limite, até que houvesse um decaimento na razão de degradação.

Este fato confirma a existência de uma dosagem ótima de $\mathrm{H}_{2} \mathrm{O}_{2}$ em torno de $20 \mathrm{mM}$. Uma concentração de peróxido de hidrogênio superior a este valor causa uma diminuição na velocidade da reação, reduzindo a razão de degradação. Para os dois tipos de corantes avaliados é observada uma redução do $\mathrm{K}\left(\mathrm{min}^{-1}\right)$ com o aumento da concentração de $\mathrm{H}_{2} \mathrm{O}_{2}$ de 20 para $30 \mathrm{mM}$. Os resultados apresentados estão em concordância com outros trabalhos relacionados ao excesso de $\mathrm{H}_{2} \mathrm{O}_{2}$. Poon et al. $^{7}$ avaliaram um modelo cinético na degradação de corantes reativos e puderam concluir que, quando o $\mathrm{H}_{2} \mathrm{O}_{2}$ estava presente em excesso no meio reacional, este apresentava pouco efeito sob a remoção do corante, sendo observada uma diminuição do $\mathrm{k}\left(\mathrm{min}^{-1}\right)$ obtido. A avaliação do modelo cinético permite confirmar a afirmativa anterior, que relaciona excesso de $\mathrm{H}_{2} \mathrm{O}_{2}$ com produção do radical hidroperoxil $\left(\mathrm{HO}_{2}{ }^{\bullet}\right)$, de capacidade reativa inferior ao radical hidroxila $(\mathrm{HO} \bullet)$.

A descoloração das soluções está associada ao rompimento das ligações azo $(\mathrm{N}=\mathrm{N})$. Para verificar se a degradação da cor poderia estar gerando carga orgânica foram realizadas análises de Demanda Química de Oxigênio (DQO). A Tabela 4 apresenta os resultados da DQO das soluções testadas. Os resultados comprovam a ocorrência de degradação da matéria orgânica.

Tabela 4. Valores de DQO para as amostras dos corantes reativos, antes e depois do tratamento com $\mathrm{H}_{2} \mathrm{O}_{2} / \mathrm{UV}\left(\mathrm{H}_{2} \mathrm{O}_{2}=20 \mathrm{mM}\right)$

\begin{tabular}{lcc}
\hline Corante & DQO $_{\text {inicial }}$ & DQO $_{\text {final }}$ \\
\hline Azul Marinho Drimarene X-GN 150 & $121,5 \mathrm{mg} / \mathrm{L}$ & $6,2 \mathrm{mg} / \mathrm{L}$ \\
Vermelho Drimarene X-6BN 150 & $121,2 \mathrm{mg} / \mathrm{L}$ & $6,0 \mathrm{mg} / \mathrm{L}$ \\
\hline
\end{tabular}

Uma redução de DQO acima de $90 \%$ pôde ser observada, comprovando que além da ruptura do grupamento cromóforo, indicado pela remoção de cor nas soluções, o sistema $\mathrm{H}_{2} \mathrm{O}_{2} / \mathrm{UV}$ é capaz de reduzir corantes reativos em intermediários facilmente degradados a $\mathrm{CO}_{2}, \mathrm{H}_{2} \mathrm{O}$ e sais inorgânicos. 


\section{CONCLUSÕES}

O uso combinado de peróxido de hidrogênio e radiação ultravioleta mostrou-se efetivo para remoção de cor em soluções de corantes reativos. Os resultados mostraram que a concentração de $\mathrm{H}_{2} \mathrm{O}_{2}$ de $20 \mathrm{mM}$ é eficaz para descolorir os corantes reativos Azul Marinho Drimarene X-GN150 e Vermelho Drimarene X-6BN 150. O uso de uma dosagem de peróxido de hidrogênio superior a este valor não apresentou resultados efetivos. Nossos resultados comprovaram que o modelo cinético de pseudo-primeira ordem está de acordo com os dados experimentais

Os resultados também indicaram que os radicais livres $\mathrm{OH} \bullet$ são capazes de oxidar o grupamento azo $(\mathrm{N}=\mathrm{N})$, reduzindo a cor e a concentração dos corantes reativos na solução, além de oxidar a carga orgânica gerada, reduzindo a Demanda Química de Oxigênio em valores acima de $90 \%$.

\section{REFERÊNCIAS}

1. Cisneros, R. L.; Espinoza, A. G.; Litter, M. I.; Chemosphere 2002, 48, 393.

2. Kurbus, T.; Slokar, Y. M.; Marechal, A.M.; Dyes Pigm. 2002, 54, 67.

3. Galindo, C.; Kalt, A.; Dyes Pigm. 1999, 42, 199.

4. Pagga, U.; Brown, D.; Chemosphere 1986, 15, 479.

5. Ganesh, R.; Boardman, G. G.; Michelsen, D.; Water Res. 1994, 28, 1367.

6. Swaminathan, K.; Sandhya, S.; Carmalin-Sophia, A.; Pachhade, K.; Subrahmanyam, Y.V.; Chemosphere 2003, 50, 619.

7. Poon, C. S.; Huang,Q.; Fung, P. C; Chemosphere 1999, 38, 1005.

8. Georgiou, D.; Medelis, P.; Aivasidis, A.; Gimouhopoulos, K.; Dyes Pigm. 2002, 52, 69 .

9. Bertazolli, R. ; Pelegrini, R.; Quim. Nova 2002, 25, 477.

10. Alaton, I. A.; Balcioglu, I. A.; Bahnemann, D. W.; Water Res. 2002, 36, 1143.

11. APHA - AWWA - WEF; Standard Methods for the Examination of Water and Wastewater, $20^{\text {th }}$ ed., New York: U.S.A, 1998.

12. Neamtu, N.; Siminiceanu, I.; Yediler, A.; Kettrup, A.; Dyes Pigm. 2002, $53,93$. 